Disclosure of Interests: None declared DOI: 10.1136/annrheumdis-2020-eular.5194

\section{AB1094 SCAR IMAGING ECHOCARDIOGRAPHY WITH ULTRASOUND MULTI-PULSE SCHEME [ESCAR] FOR THE DETECTION OF MYOCARDIAL FIBROSIS IN PATIENTS WITH SYSTEMIC LUPUS ERYTHEMATOSUS PRELIMINARY RESULTS.}

A. Giollo ${ }^{1}$, G. Vinco ${ }^{2}$, G. Orsolini ${ }^{1}$, G. Cioffi ${ }^{1}$, G. Adami ${ }^{1}$, A. Fassio ${ }^{1}$, L. Idolazzi ${ }^{1}$, D. Gatti ${ }^{1}$, F. L. Ribichini ${ }^{2}$, M. Rossini ${ }^{1}$, O. Viapiana ${ }^{1} .{ }^{1}$ University of Verona, Rheumatology Section, Department of Medicine, Verona, Italy; ${ }^{2}$ University of Verona, Cardiology Unit, Department of Medicine, Verona, Italy

Background: Myocardial fibrosis is a severe complication of immune-mediated diseases, occurring in up to $30 \%$ of systemic lupus erythematosus (SLE) patients. Cardiovascular magnetic resonance imaging allows myocardial scar detection in SLE patients, but it is costly, time consuming, and unfit for patients with rena disease. Scar imaging echocardiography with ultrasound multi-pulse scheme (eSCAR) is a novel and promising technique that proved to be effective in detecting ischemic myocardial scars in patients with coronary artery disease (CAD). Objectives: To evaluate if the eSCAR technique is feasible and to better characterize SLE patients with cardiac involvement by eSCAR.

Methods: We recruited consecutive patients with SLE classified according to the 2019 EULAR/ACR recommendations. Patients with diabetes mellitus, obesity, prior cardiovascular (CV) disease or anti-phospholipid (aPL) syndrome were excluded. Eligible participants underwent a thorough clinical assessment and a full echocardiography examination, including the eSCAR technique. Data on clinical variables were collected; disease activity was estimated by the SLE Disease Activity Index (SLEDAI) score. Lupus flare was defined as new/worse clinical signs and symptoms and/or lab measurements and a change/increase in treatment. Patients were compared according to the presence or absence of eSCAR In this preliminary report, only descriptive analyses are provided. Continuous data are reported as median $\left[25^{\text {th }} ; 75^{\text {th }}\right.$ percentile].

Results: We enrolled fifteen patients diagnosed with SLE (age 45 years [36; 47], disease duration 14 years [12; 20]), 13 (87\%) were females. Median SLEDAI was 5 [2; 8]. The most frequent disease involvement included arthritis (73\%), skin and mucous membranes $(60 \%)$, lupus nephritis $(47 \%)$ and cytopenias $(47 \%)$. Patients had received treatment for lupus with 5 drugs $[5 ; 8]$ Cumulative prednisone dosage was $25 \mathrm{~g}$ [20; 44], whilst the current daily dosage of prednisone was $4 \mathrm{mg}[0.0 ; 5.0]$. Hypertension was present in $4(27 \%)$ and hypercholesterolemia in $2(13 \%)$ subjects; 4 patients $(27 \%)$ were current or past smokers. The eSCAR technique was feasible in all participants with no adverse effects. Myocardial scars were detected in 2 patients (eSCAR-positive $13 \%$; figure and table); eSCAR positive patients were females and had no history of cardiovascular involvement (including pericarditis); they had at least one relapse within the prior 12 months before enrollment; at least one cardiovascular risk factor was found in both patients (one was a smoker and the other one had hypertension); none received prior treatment with cyclophosphamide or rituximab; they had no renal involvement; arthritis and cytopenia were the prominent features of disease; anti-dsDNA titer was higher than eSCAR-negative patients.

Conclusion: Echocardiography allowed detection of myocardial scars in patients with SLE. Our preliminary data show that eSCAR is feasible and well tolerated in a SLE population. Further data from this ongoing study will help investigate whether eSCAR might improve risk stratification, by identifying myocardial involvement in SLE patients with a more active disease.

Table

\begin{tabular}{lccc}
\hline & $\begin{array}{c}\text { eSCAR positive } \\
\text { Patient 1 }\end{array}$ & $\begin{array}{c}\text { eSCAR positive } \\
\text { Patient 2 }\end{array}$ & $\begin{array}{c}\text { eSCAR negative } \\
\text { SLE patients ( } \mathrm{n}=13)\end{array}$ \\
\hline Age, years & 32 & 45 & 45 \\
Sex & Female & Female & $11 \mathrm{~F} / 2 \mathrm{M}$ \\
ESR mm/h & 29 & 7 & 15 \\
CRP mg/L & 3 & 0.6 & 2 \\
Anti-dsDNA (IF) & Positive & Positive & Positive 7 (47) \\
Anti-dsDNA (CLIA) & 105 & 149 & $34[7 ; 66]$ \\
C3 (mg/L) & 61 & 80 & $85[70 ; 94]$ \\
C4 (mg/L) & 4 & 13 & $10[9 ; 16]$ \\
P-Cr (mg/dL) & 0.6 & 0.8 & $0.7[0.6 ; 0.7]$ \\
aPL & Positive & Negative & $7(47)$ \\
Disease duration, years & 15 & 28 & $14[12 ; 15]$ \\
SLEDAl & 12 & 0 & $5[2 ; 8]$ \\
Lupus flare in the past 12 months & yes & yes & $5(33)$ \\
Cumulative prednisone dosage (g) & 60 & 61 & $24[19,32]$ \\
Previous use of cyclophosphamide & No & No & $3(20)$ \\
Previous use of rituximab & No & No & $1(1)$ \\
\hline
\end{tabular}
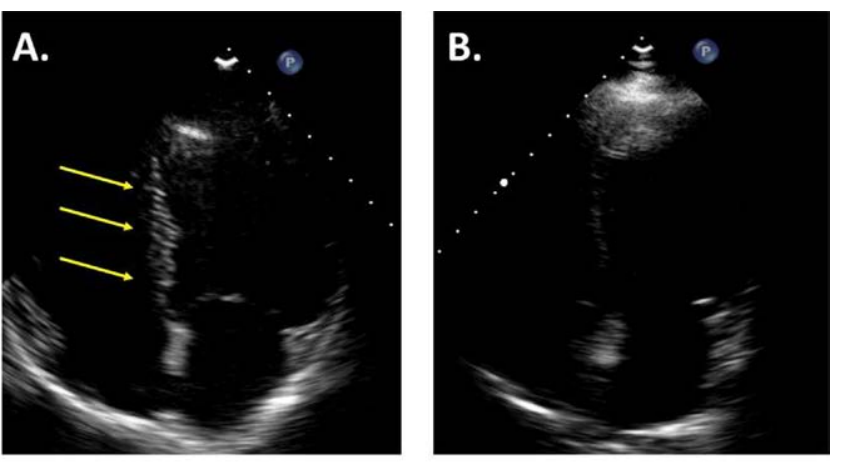

Figure. A. Myocardial fibrosis in the interventricular septum (eSCAR-positive patient). B. no myocardial fibrosis (eSCAR-negative patient)

Acknowledgments: This study was granted by Gruppo LES Italia Onlus Disclosure of Interests: Alessandro Giollo: None declared, Giulia Vinco: None declared, Giovanni Orsolini: None declared, Giovanni Cioffi: None declared, Giovanni Adami: None declared, Angelo Fassio Speakers bureau: Angelo Fassio reports personal fees from: Abiogen and Novartis, outside the submitted work., Luca Idolazzi: None declared, Davide Gatti Speakers bureau: Davide Gatti reports personal fees from Abiogen, Amgen, Janssen-Cilag, Mundipharma, outside the submitted work., Flavio Luciano Ribichini: None declared, Maurizio Rossini Speakers bureau: AbbVie, Abiogen, Amgen, BMS, Eli-Lilly, Novartis, Pfizer, Sanofi, Sandoz and UCB, Ombretta Viapiana: None declared

DOI: 10.1136/annrheumdis-2020-eular.5006

\section{AB1095 DIAGNOSTIC PERFORMANCE OF THE AORTIC WALL THICKENING DETERMINED BY ROUTINE CT-SCAN FOR THE DIAGNOSIS OF AORTITIS OVER 50 YEARS OF AGE.}

G. Grouteau ${ }^{1}$, F. Lafourcade ${ }^{1}$, H. Fayolle ${ }^{1}$, C. Ricard ${ }^{1}$, L. Balardy ${ }^{1}$, P. Delobel ${ }^{1}$, A. Constantin ${ }^{1}$, O. Beyne Rauzy ${ }^{1}$, L. Alric ${ }^{1}$, P. Payoux ${ }^{1}$ A. Hitzel ${ }^{1}$, M. Faruch ${ }^{1}$, L. Sailler ${ }^{1} .{ }^{1}$ University Hospital De Toulouse, Toulouse, France

Background: Making the diagnosis of aortitis may be challenging in the "real life" ${ }^{18}$ F-FDG PET/CT (PET) has emerged has the gold standard to diagnose aortitis but it is expensive and not always quickly available. Injected CT-scan (CT) can also be used to diagnose aortitis, which is suspected when there is an extended circumferential, non-atherosclerotic increase of the aortic wall thickness (AWT) However, data are lacking on the diagnostic performances of AWT to diagnose aortitis.

Objectives: To describe the diagnostic performance of AWT measured by CT to diagnose aortitis in patients over 50 years of age.

Methods: We performed a monocentric retrospective study between 2013 and 2018 including 1) all patients over 50 years of age who had performed a PET for inflammation or fever of unknown origin or for suspicion of classical giant cell arteritis (GCA), aortitis or large vessel vasculitis (LVV); 2) with an injected CT-scan performed in the 30 days before or after PET; 3 ) not diagnosed to have cancer or infection 4) exposed to corticosteroids for less than 3 days at the time of CT and PET. The gold standard for aortitis was PET positivity according to the 2018 European consensus grading criteria (1). AWT was measured at differen aortic segments (ascending aorta, descending thoracic aorta and suprarenal abdominal aorta) by a single radiologist unaware of the result of PET and of the previous interpretation of CT-scan.

Results: Eighty-nine patients were included (female: 47\%; mean age 68.8 (5093) years; GCA: $n=28$ ). Twenty patients had aortitis according to the PET result (GCA: $n=16$ ).

Mean maximal aortic wall thickening was: $3.25 \mathrm{~mm}$ (median: 3.3; range: 2-4.6) in the aortitis group and $2.2 \mathrm{~mm}$ (median: 2.1 ; range $1-3.8$ ) in the negative PET group $(\mathrm{p}<0.001)$

The best ROC curve AUC ( $85 \%$ ) was obtained considering the maximal AWT on the thoracic and suprarenal abdominal aorta only. The AUC of the ROC curve at the ascendant thoracic aorta was poor (75\%) (Figure).

The Youden's index of the ROC curve was $2.6 \mathrm{~mm}$ with a sensitivity of $85 \%$ and specificity of $71 \%$ (Table) for aortitis diagnosis. An AWT $\leq 2.0 \mathrm{~mm}$ exclude the diagnosis of aortitis. The $3 \mathrm{~mm}$ threshold had a PPV of only $52 \%$ and an AWT $\geq$ $4 \mathrm{~mm}$ was fully specific.

Conclusion: Aortitis diagnosis using a routine injected CT-scan interpreted by a single radiologist may be certain when showing an AWT $\geq 4$ and excluded for an AWT $\leq 2.0$. The threshold of $3 \mathrm{~mm}$ seems not sufficiently specific to diagnose aortitis without a confirmatory PET. 
References:

[1] Riemer H.J.A. Slart et al. FDG-PET/CT(A) imaging in large vessel vasculitis and polymyalgia rheumatica: joint procedural recommendation of the EANM, SNMMI, and the PET Interest Group (PIG), and endorsed by the ASNC. Eur J Nucl Med Mol Imaging. 2018 Jul;45(7):1250-69.

Table. Performance of different threshold for maximal thickness of the aortic wall for diagnosis of aortitis using PET positivity as the gold standard*.

\begin{tabular}{llllll}
\hline Thickness $(\mathrm{mm})$ & Sensitivity & Specificity & NPV & PPV & accuracy \\
\hline$\geq 1.9$ & $100 \%$ & $30.4 \%$ & $100 \%$ & $29.41 \%$ & $46 \%$ \\
$\geq 2.0$ & $100 \%$ & $31.88 \%$ & $100 \%$ & $29.85 \%$ & $47 \%$ \\
$\geq 2.2$ & $95 \%$ & $53.62 \%$ & $97.36 \%$ & $37.25 \%$ & $63 \%$ \\
$\geq 2.6$ & $85 \%$ & $71.01 \%$ & $94.23 \%$ & $45.94 \%$ & $74 \%$ \\
$\geq 3.0$ & $60 \%$ & $84.05 \%$ & $87.88 \%$ & $52.17 \%$ & $78 \%$ \\
$\geq 3.3$ & $60 \%$ & $92.75 \%$ & $88.89 \%$ & $70.59 \%$ & $85 \%$ \\
$\geq 3.5$ & $35 \%$ & $95.65 \%$ & $83.54 \%$ & $70 \%$ & $82 \%$ \\
$\geq 3.8$ & $25 \%$ & $98.55 \%$ & $81.92 \%$ & $83.33 \%$ & $82 \%$ \\
$\geq 4.0$ & $20 \%$ & $100 \%$ & $81.17 \%$ & $100 \%$ & $82 \%$
\end{tabular}

${ }^{*}$ Made with ROC curve in descendant thoracic and suprarenal segment of the aorta; PET: ${ }^{18} \mathrm{~F}$ FDG PET/CT; NPV: negative predictive value; PPV: positive predictive value.

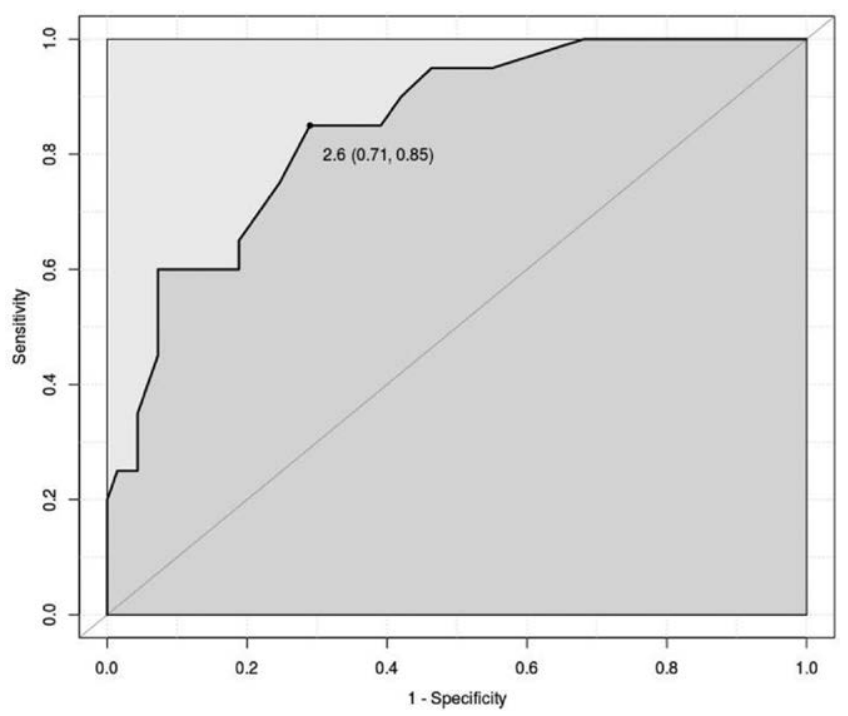

Figure. ROC curve of aortic wall thickness, in descendant thoracic and suprarenal abdominal segments for the diagnosis of aortitis.

Disclosure of Interests: None declared

DOI: 10.1136/annrheumdis-2020-eular.2804

\section{AB1096 AND PATIENT REPORTED OUTCOMES IN SPONDYLOARTHRITIS AND PSORIATIC ARTHRITIS: A SYSTEMATIC LITERATURE REVIEW}

T. Gudu' 1 , M. A. D'agostino ${ }^{1} .{ }^{1}$ Ambroise Pare Hospital, Rheumatology, Boulogne-Billancourt, France

Background: Patient reported outcomes (PROs) reflect patients' opinion on disease activity and impact of disease on various aspects of life. They are essential in the assessment of patients spondyloarthritis (SpA) and psoriatic arthritis (PsA), but may be influenced by several factors other than disease activity and severity. Ultrasound (US) is an objective tool to evaluate inflammation and structural damage in these conditions.

Objectives: The objective of this study was to determine if and to which extent the US assessment reflects patient perspective in patients with SpA and PsA.

Methods: Two separate systematic literature reviews were conducted for each disease. The research questions were formulated according to the PICO framework. The patient reported domains of health were selected from the ones included in the Core Set for SpA [1] and PsA [2] and from the ones frequently reported in clinical trials and observational studies for SpA and PsA, as well as patient self-assessment of pain at the joint level. We
Table 1. Patient reported domains evaluated in the included studies.

\begin{tabular}{llc}
\hline & Domain & Studies reporting the domain \\
& $\mathrm{N}(\%)$ \\
\hline SpA & Disease activity & $11(91.7)$ \\
& Function/ disability & $9(75)$ \\
& Pain & $7(58.3)$ \\
& Patient global assessment & $5(41.7)$ \\
Quality of life & $5(41.7)$ \\
Morning stiffness & $3(25)$ \\
Fatigue & $1(8.3)$ \\
Sleep & $1(8.3)$ \\
Global health & $1(8.3)$ \\
Patient reported peripheral swelling & $1(8.3)$ \\
Function/ disability & $10(83.3)$ \\
Pain & $9(75)$ \\
Patient global assessment & $8(66.7)$ \\
Disease activity & $5(41.7)$ \\
Quality of life & $4(33.3)$ \\
Disease impact & $3(25)$ \\
Mood disorders & $2(16.7)$ \\
Fatigue & $2(16.7)$ \\
Morning stiffness & $1(8.3)$
\end{tabular}

included articles that evaluated any kind of relationship between PROs and US assessment.

Results: $\mathrm{SpA}$

In total, 2977 abstracts were identified, of which 12 articles were retained in the final analysis: 10 cross-sectional studies assessing disease activity state and 2 longitudinal, non-interventional trials assessing treatment response. The most frequently evaluated domains of health are depicted in table 1.

The cross-sectional studies evaluating only one joint area (foot and ankle, sacroiliac joint, shoulder and hip) showed that overall there is no important association between the PROs and the US parameters. In studies evaluating multiple entheses sites there were several correlations between PROs (pain, disease activity, function, quality of life (QoL)) and US variables, i.e., US inflammatory scores and total enthesitis scores, comprising both inflammatory and structural changes $(r=0.24-0.53)$. In the longitudinal studies there was no consistent association between PROs and US variables.

PsA

Out of the 1267 abstracts identified, 12 were finally included in the qualitative analysis: 5 cross-sectional studies on disease activity state and 7 longitudinal studies, of which 2 on disease activity state and 5 on treatment response. The most frequently evaluated domains of health are depicted in table 1

Overall, in the cross-sectional studies, there was no consistent association between PROs and US assessment. In the longitudinal studies on disease activity, pain and patient global assessment (PGA) were associated with US inflammatory variables $(r=0.22-0.28)$, while disability correlated with US damage $(r=0.22-0.39)$. Change in PGA and pain was associated with change in the global US inflammation score $(r=0.28-0.35)$. In longitudinal studies on treatment response results were inconsistent: two studies showed that there was significant parallel trend of some PROs (function, pain and PGA) and some US entheses scores, but the other 3 studies showed no significant association.

Conclusion: The association between PROs and US in SpA and PsA was rather inconsistent. Patient perspective and US examination seem to provide different, but complementary perspectives on disease assessment and both should be taken into account in SpA and PsA management.

References:

[1] van der Heijde, J Rheumatol 1999

[2] Orbai, An Rheum Dis 2016

Acknowledgments: Supported by PARTNER Fellowship

Disclosure of Interests: None declared

DOI: 10.1136/annrheumdis-2020-eular.4316

\section{\begin{tabular}{|l|l}
\hline AB1097 THE ASSOCIATION OF ULTRASOUND ASSESSMENT \\
\hline
\end{tabular} AND PATIENT REPORTED OUTCOMES IN RHEUMATOID ARTHRITIS: A SYSTEMATIC LITERATURE REVIEW}

T. Gudu' ${ }^{1}$, M. A. D'agostino' ${ }^{1}$ Ambroise Pare Hospital, Rheumatology, Boulogne-Billancourt, France

Background: Patient reported outcomes (PROs) are recognized to be essential outcome measures in the assessment of patients with rheumatoid arthritis (RA), but they can be prone to being influenced by multiple variables. Thus, objective 